\title{
Three-Dimensional Reconstruction of the Semicircular Canals with a Two-Hands Model
}

\author{
Original Investigation $>\begin{aligned} & \text { Rasim Y1 } \text { Ymazer }^{1} \text { (D), Osman Melih Topçuoğlu }{ }^{2} \text { (D) } \\ & \text { Department of Otorhinolaryngology, Demiroğlu Bilim University School of Medicine, İstanbul, Turkey }\end{aligned}$ \\ ${ }^{2}$ Department of Radiology, Yeditepe University School of Medicine, İstanbul, Turkey
}

Abstract

ORCID IDs of the authors:

R.Y. 0000-0002-2447-2446;

O.M.T. 0000-0002-4008-3395.

Cite this article as: Yılmazer R, Topçuoğlu OM. Three-Dimensional Reconstruction of the Semicircular Canals with a Two-Hands Model. Turk Arch Otorhinolaryngol 2019; 57(4): 176-81.

\section{Corresponding Author:}

Rasim Yılmazer; ryno78@gmail.com

Received Date: 08.06.2019 Accepted Date: 24.09 .2019

Content of this journal is licensed under a Creative Commons Attribution 4.0 International License. Available online at www.turkarchotolaryngol.net
Objective: To help understand the anatomy and the diseases of the semicircular canals (SCCs) by defining the three-dimensional reconstruction of the SCCs in a model reconstructed using the two hands (two-hands model) and to determine the three-dimensional configuration of the two hands by measuring the angle between the SCCs and the sagittal plane.

Methods: Patients older than 18 years of age with computed tomography (CT) scans of the temporal bones taken between 2017 and 2018 at the Yeditepe University Hospital were included in the study. CT images were evaluated by a radiologist. The angles between every SCC and the sagittal plane were measured with the MIMICS 12.1 program. Mean angles between the SCCs and the sagittal plane were mea- sured. These angles were demonstrated on the twohands model of the SCCs by means of a goniometer and photographed.

Results: Thirty patients and 60 temporal bones were included in the study. Mean angles between the superior, the posterior, and the horizontal SCCs and the sagittal plane were found as $37.1 \pm 3.3^{\circ}, 50.4 \pm 4.1^{\circ}$, and $89.5 \pm 4.7^{\circ}$, respectively.

Conclusion: This is the first study to define the three-dimensional representation of the SCCs with a two-hands model. This may allow for a better understanding and the better teaching of the anatomy and diseases of SCCs.

Keywords: Semicircular canals, anatomy, anatomic model, inner ear, benign paroxysmal positional vertigo

\section{Introduction}

The anatomy of the semicircular canals (SCCs) is particularly difficult to understand as it requires three-dimensional (3D) conception of their complex geometry. Benign paroxysmal positional vertigo (BPPV), a disease of the vestibular end-organs such as the SCCs and the utricle, is the most common cause of peripheral vertigo (1). Knowledge of the anatomy of the semicircular canals and their position relative to the head is crucial to understand the pathophysiology, diagnostic workup and treatment of BPPV. To date, virtual and printed 3D models of the SCCs have been made to improve anatomy education and to explain the principals of diagnosis and treatment of BPPV (2-5). However, these new 3D models are not available in most of the medical schools and ENT clinics because extra equipment, such as a $3 \mathrm{D}$ printer and a computer, are required and/or the cost of these models makes it difficult to get them. Therefore, a cheap, practical, readily available model of the SCCs that does not require additional equipment is needed.

In this study, we defined a three-dimensional reconstruction of the SCCs in a model created using hand gestures (two-hands model). To the best of our knowledge, a hand model of the SCCs has not been previously defined in the literature. We also aimed to determine the three-dimensional configuration of the two hands by measuring the angle between the SCCs and the sagittal plane. 


\section{Methods}

\section{Subjects}

Patients older than 18 years of age with computed tomography (CT) scans of the temporal bones taken at the Yeditepe University Hospital between January 2017 and December 2018 were included in the study. Patients with an inner ear anomaly, a history of temporal bone fracture, and absent or traumatized SCCs due to a congenital anomaly or surgery such as labyrinthectomy were excluded. CT images of 30 patients and 60 temporal bones retrieved from the hospital's radiology Picture Archiving and Communication System (PACS) were evaluated. None of the patients were excluded from the study at this stage. The study protocol was approved by the Yeditepe University School of Medicine Ethics Committee (Ethics Committee No: 2018/1518). A written informed consent was obtained from each patient. The study was conducted in line with the principles of the Declaration of Helsinki. CT images of bilateral temporal bones were assessed by one radiologist with a neuroradiology experience of eight years.

All CT images were obtained with 64-detector multi-slice CT (Revolution HD/GSI, GE Healthcare, Chalfont St Giles, UK). The scanning technique was the standard temporal bone CT imaging protocol using tube voltage of $120 \mathrm{kVp}$, tube current of $180 \mathrm{~mA}$, slice thickness of $0.625 \mathrm{~mm}$, matrix of $512 \times 512$ and FOV of $22 \mathrm{~cm} \times 22 \mathrm{~cm}$. The images were reconstructed in a bone window.

\section{Coordinates and Measurements}

Raw data included in CT images show the spatial localizations in the cranio-caudal, antero-posterior and in the left to right directions. Three-dimensional orientations of the semicircular canals with respect to the sagittal plane require head-based co-

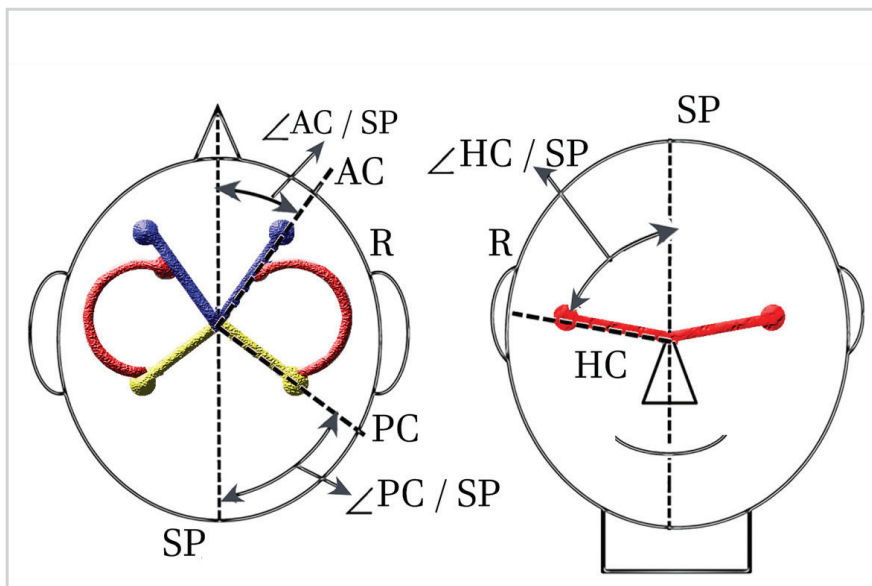

Figure 1. The angles between the semicircular canal planes and the head mid-sagittal plane (right ear)

$\angle \mathrm{AC} / \mathrm{SP}$ : angle between the anterior canal $(\mathrm{AC})$ plane and the sagittal plane (SP). $\angle \mathrm{PC} / \mathrm{SP}$ : angle between the posterior canal (PC) plane and the SP. $\angle \mathrm{HC} / \mathrm{SP}$ : angle between the horizontal canal (HC) plane and the SP. Reprinted and adapted with permission (7) ordinates. The images were assessed in a dedicated program of MIMICS Medical 12.1 (Materialize,Leuven, Belgium) and the angles between the SCCs and the mid-sagittal plane were measured.The angles measured in $\mathrm{CT}$ analyses are shown in Figure 1. $\angle \mathrm{AC} / \mathrm{SP}$ is the angle between the anterior SCC and the Psag. $\angle \mathrm{HC} / \mathrm{SP}$ is the angle between the horizontal $\mathrm{SCC}$ and the Psag. $\angle \mathrm{PC} / \mathrm{SP}$ is the angle between the posterior $\mathrm{SCC}$ and the Psag. The anatomical landmarks of the temporal bones were assessed using the MIMICS program. To accurately calculate the orientations of the SCCs with respect to the skull base, we used a standard three-dimensional system which was published by the Frankfort horizontal plane (Pfrkt) (6). This system was independent from the position of the head during the $\mathrm{CT}$ scanning. The Pfrkt plane is characterized to pass through the right inferior orbital border and bilateral superior borders of external auditory canals. The mid-sagittal plane was described as a plane passing through the midpoint of the anterior foramen magnum and the center of the line connecting the tips of the two posterior clinoid processes lying perpendicular to the Pfrkt plane (Figure 2). Additionally, crista galli was used as a third landmark because the program requires three points to create a plane. The SCC planes were defined by three anatomical regions: the ampulla-side end, the non-ampulla-side end and their midpoints for each canal (7). Then, the angle between the sagittal plane and the SCC planes were calculated by means of the described model (8). The orientation of the SCCs in the skull was defined by the angles between each of the canals and the standard mid-sagittal reference plane. Measurement of the angle between the posterior SCC plane and the sagittal plane is shown in Figure 3.

\section{The Two-Hands Model}

The superior view of the three-dimensional model of the right SCCs was created with two hands as follows (Figure 4): With

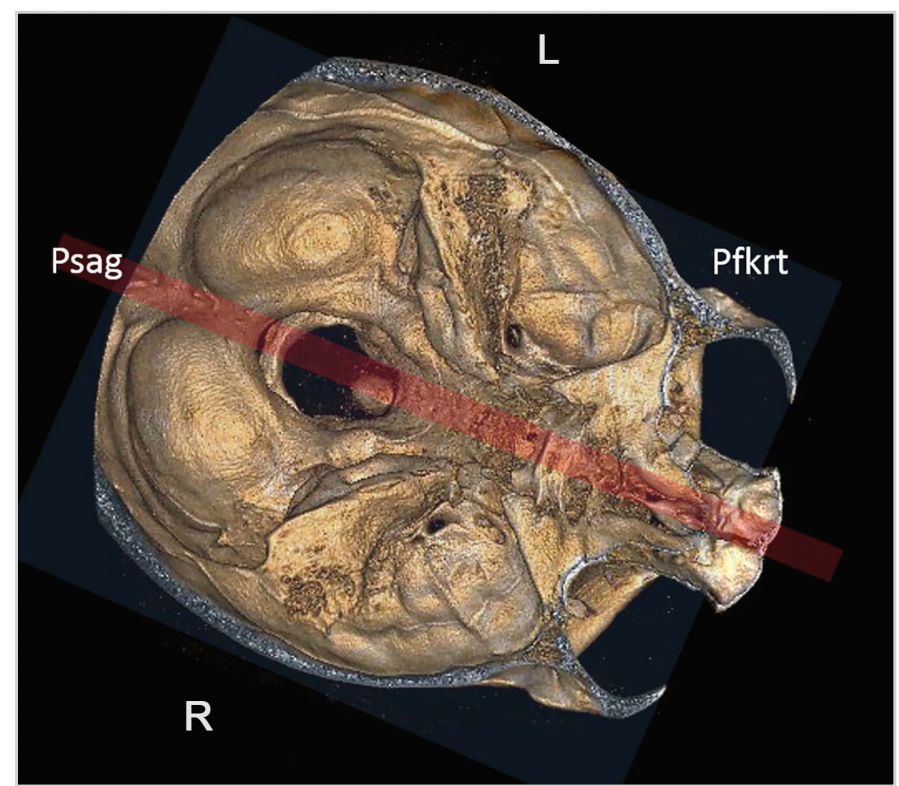

Figure 2. Axial cut of the skull showing the mid-sagittal plane (Psag) which is perpendicular to the Frankfort horizontal plane (Pfkrt) 
the left hand in pronation, the thumb and the index finger were shaped as a semicircle, with the tips of these two digits touching at a $90^{\circ}$ angle. Then, with the right hand in pronation, the thumb and index finger of the right hand were shaped as a semicircle, the tip of the index finger perpendicularly touching the ulnar side of the first metacarpophalangeal joint of the pronated left hand. At the same time, the tip of the thumb perpendicularly touched the radial side of that joint. In this model, the index finger of the left hand represents the superior

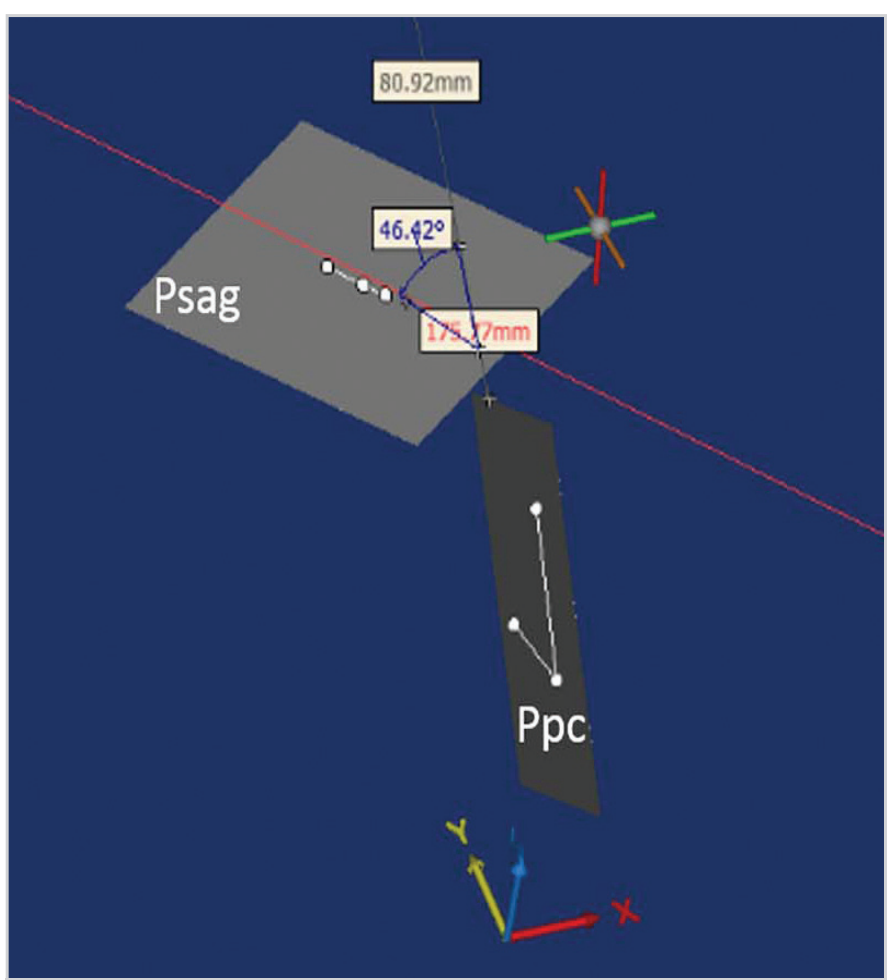

Figure 3. The measurement of the angle between the left posterior semicircular canal plane (Ppc) and the mid-sagittal plane (Psag). The red line shows the mid-sagittal line. The blue line shows the direction of the Ppc
SCC, the thumb of the left hand represents the lateral (horizontal) SCC, and the thumb and the index finger of the right hand represent the posterior SCC. The nails of the left index finger, the left and the right thumbs represent the ampullae of the SCCs. In order to both create the common crus and hide the nail which does not represent an ampulla, the nail of the right index finger is abutted to the skin between the thumb and the index finger of the left hand. The left metacarpophalangeal joint represents the utricle. The third, fourth and fifth fingers of the left hand represent the lateral orientation of the apex of the cochlea. The two hands were reversed for the 3D model of the left SCCs. To exemplify, Figures 4B and 4C show the two-hands models for the posteromedial view of the $3 \mathrm{D}$ model of the right SCCs and the multiplanar $\mathrm{CT}$ reconstruction of the human labyrinth, respectively. The mean angles between the SCCs and the sagittal plane were demonstrated on the two-hands model of the SCCs by means of a goniometer and photography.

\section{Statistical analysis}

Statistical analyses were performed using the Statistical Package for Social Sciences sofware version 25.0 (IBM Corp.; Armonk, NY, USA). Descriptive analyses were presented with mean, standard deviation, minimum and maximum values for continuous data, and with frequencies and percentages for categorical data. The variables were investigated using the Kolmogorov-Smirnov test to explore whether they were normally distributed. The Mann-Whitney $U$ test was used to compare the differences between genders. The Mann-Whitney $U$ test was also used to compare the differences between right and left sides of $\angle \mathrm{AC} / \mathrm{SP}$ and $\angle \mathrm{HC} / \mathrm{SP}$. Two independent samples t-test was used to compare the differences between right and left sides of $\angle \mathrm{PC} / \mathrm{SP}$. Relationships between age and measurements were determined using the Pearson's Correlation Coefficients. A 5\% 0.05 type-I error level was used to determine statistical significance.
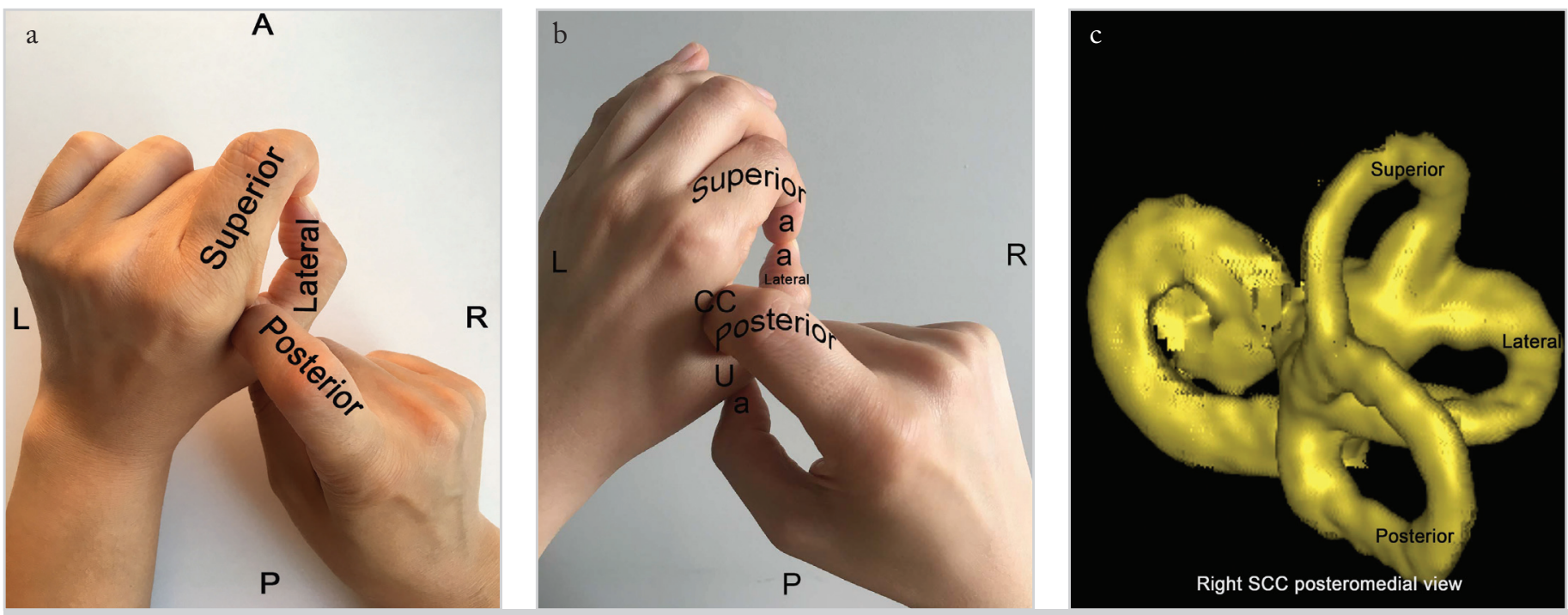

Figure 4. a-c. Superior view of the two-hands model of the right semicircular canals (a). Posteromedial view of the two-hands model of the right semicircular canals (b). Three-dimensional reconstructed computed tomography image shows the right semicircular canals from posteromedial view (c) A: anterior, P: posterior, R: right, L: left, R: right, a: ampulla, CC: common crus, U: utricle, SCC: semicircular canal 
Table 1. Descriptive statistics of the angle measurements between each SCC and the head mid-sagittal plane

\begin{tabular}{l|l|c|c|c|c}
\hline Angles & N & Minimum & Maximum & Mean & Standard deviation \\
\hline $\mathrm{R}-\angle \mathrm{HC} / \mathrm{SP}$ & 30 & 69.0 & 102.6 & 90.1 & 6.7 \\
\hline $\mathrm{L}-\angle \mathrm{HC} / \mathrm{SP}$ & 30 & 82.1 & 102.6 & 88.9 & 5.0 \\
\hline $\mathrm{R}-\angle \mathrm{PC} / \mathrm{SP}$ & 30 & 38.9 & 59.1 & 49.3 & 4.9 \\
\hline $\mathrm{L}-\angle \mathrm{PC} / \mathrm{SP}$ & 30 & 41.5 & 60.4 & 51.4 & 4.7 \\
\hline $\mathrm{R}-\angle \mathrm{AC} / \mathrm{SP}$ & 30 & 28.6 & 43.7 & 36.5 & 3.6 \\
\hline $\mathrm{L}-\angle \mathrm{AC} / \mathrm{SP}$ & 30 & 28.9 & 49.0 & 37.7 & 4.6 \\
\hline
\end{tabular}

SCC: semicircular canal, R: right, L: left, $\angle$ : angle, HC: horizontal canal, PC: posterior canal, AC: anterior canal, SP: sagittal plane

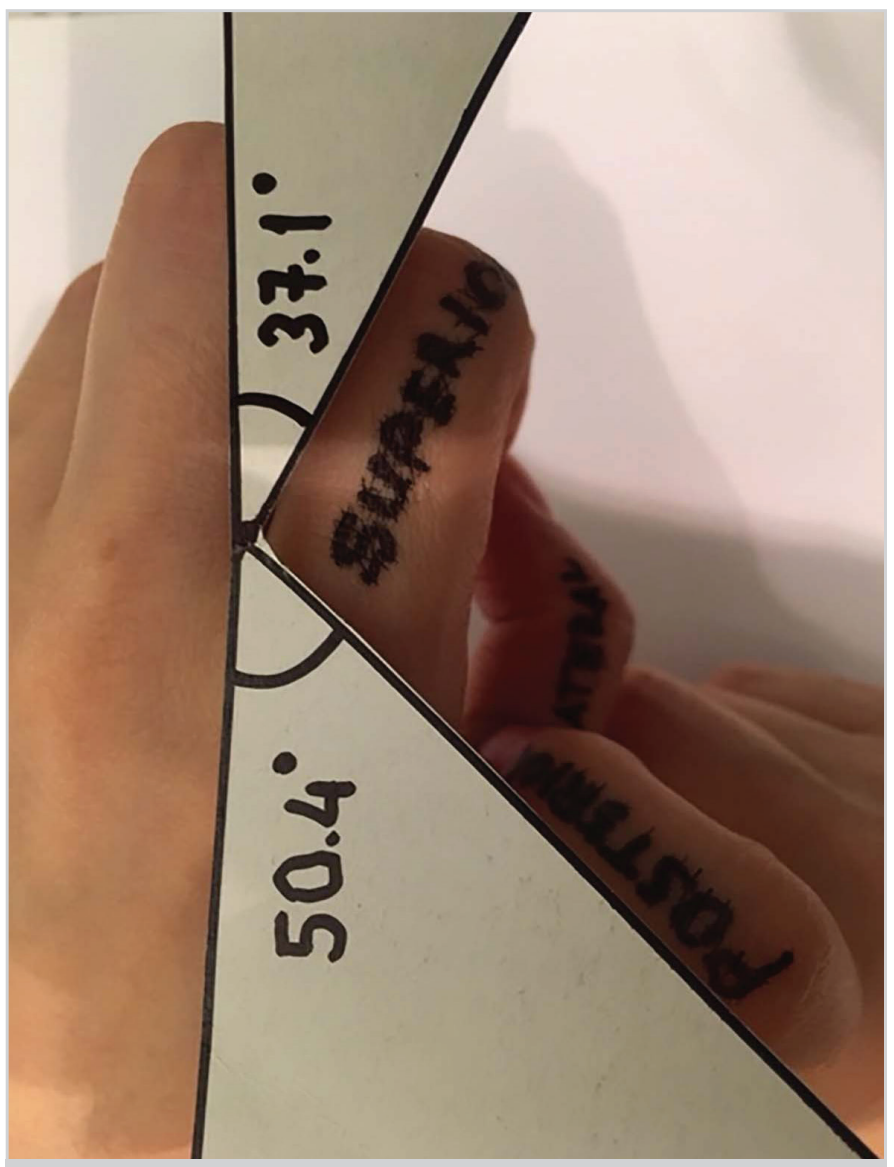

Figure 5. Superior view of the right semicircular canals shown with the two-hands model. The mean angles between the superior and the posterior semicircular canals and the sagittal plane are demonstrated

\section{Results}

Of the 30 patients included in the study 19 were male and 11 were female. Their mean age was $42.6 \pm 8.3$ years (min: 28 , max: 68). The mean angle of the anterior (superior) SCC to the head mid-sagittal plane was $37.1 \pm 3.3^{\circ}$ (min: 32.2 , $\max : 44.3$ ). The mean angle of the posterior SCC to the mid-sagittal plane was $50.4 \pm 4.1^{\circ}$ (min: 43.6, max: 58.6). The mean angle of the horizontal SCC to the mid-sagittal plane was $89.5 \pm 4.7^{\circ}$ (min: 79.7, max: 99.6). The angle measurements between each SCC and the mid-sagittal plane are shown in Table 1.

No statistically significant difference was found in terms of angle measurements based on gender and SCC side. Additionally, no relationship was found between the ages and the measurements. Figure 5 demonstrates the two-hands model of the mean angles between the superior and the posterior $\mathrm{SCC}$ s and the SPs. The angle between the horizontal SCC and the SP is not shown as it was almost a right angle.

\section{Discussion}

This 3D two-hands model of the SCCs is intended for use as a teaching tool and a quick reference guide when dealing with various pathologies of the SCCs. Although it is a very similar model of the SCCs, we cannot claim that it is a completely accurate anatomic model; it is rather a rough spatial model. Its major advantages are that it is free, simple, available in every setting, and requires no equipment. The nails of the fingers creating the SCCs show the ampullae of the SCCs. To learn and remember the locations of the ampullae can be difficult, but this model makes it easy to learn and teach. This model can also show the lateral orientation of the apex of cochlea. Rivas (4) created a multicolored cardboard model of the bilateral SCCs but the angles of the SCCs to the sagittal plane were not measured nor mentioned to show the spatial configuration of the SCCs in more detail. It is portable and has a low cost but requires to be carried wherever you need it. Teixido et al. (2) developed a virtual 3D BPPV model for study of otolith disease and a free downloadable version of this model is available. However, it would require a computer and software. A 3D-printed model of the vestibulocochlear organ within a transparent model of the temporal bone has been produced by Suzuki et al. (5) but requires a $3 \mathrm{D}$ printer which is expensive and not available in every school or clinic. There is also a medical device called DizzyFix which was developed to assist in the performance of the Epley maneuver for the treatment of posterior canal BPPV (9). It is essentially an analog of the particle repositioning maneuver rather than an analog of the semicircular canals. The device consists of a plastic tube attached to a hat. It has little resemblance to the shape of the posterior SCC but it does not show the other SCCs. It also has a high price and is not available in every clinic.

When demonstrating the 3D model of the SCCs with hand gestures, the spatial configuration of the fingers that represent the SCCs should be determined so as to create a similar model of the SCCs. The angles between each SCC, and the angles 
between the SCCs and the sagittal plane have been previously described in histological and radiological studies (10-13). Kim et al. (10) investigated the angles and the orientation of SCCs by using three-dimensional images reconstructed from temporal bone magnetic resonance imaging and reported that each of the SCCs canals made an almost right angle with the other two canals, and the horizontal semicircular canal was tilted $30^{\circ}$ from the axial plane. Since these results are consistent with previous studies $(7,11-13)$ and acknowledged by many authors, we did not re-measure these angles. They also reported that vertical (superior and posterior) semicircular canals were $45^{\circ}$ in relation to the sagittal plane (10). We could use these results as well, but since there are different measurements in the literature, we decided to measure the angles between the SCCs and the sagittal plane. Kim et al. (10) found that the angles between the superior and the posterior SCCs and the sagittal plane were $43.0^{\circ}$ and $52.5^{\circ}$, respectively. Aoki et al. (7) reported that the angles between the superior, the posterior, and the horizontal SCCs and the sagittal plane were $34.5^{\circ}, 51.1^{\circ}$, and $90.4^{\circ}$, respectively. Hashimoto et al. (11) reported these angles as $35.0^{\circ}, 54.6^{\circ}$, and $98.2^{\circ}$, respectively. Lyu et al. (8) found these angles in adults as $26.8^{\circ}, 43.0^{\circ}$, and $89.5^{\circ}$, respectively. We found these angles to be $37.1^{\circ}, 50.4^{\circ}$, and $89.5^{\circ}$, respectively. As our results were consistent with the literature, we used our results to create the spatial configuration of the SCCs in our two-hands model. Since creating these angles in the two-hands model may be difficult, a finger goniometer can be used for more accuracy, or we can assume that vertical canals are oriented at roughly $45^{\circ}$ in relation to the sagittal plane (14).

The main limitation of our study was the small sample size. But our results were comparable to those reported in previous studies. The measurements could also have been verified by a second radiologist. The strength of our study was defining a new 3D model for the SCCs.

\section{Conclusion}

This is the first study to define the three-dimensional representation of the SCCs with a two-hands model. This may allow for a better understanding and the better teaching of the anatomy and diseases of SCCs. This model can be used to simulate the diagnostic maneuvers and the treatment procedures used for BPPV. It has great potential as a teaching tool and can offer insights that may lead to more accurate diagnosis and treatment of BPPV.

Ethics Committee Approval: Ethics committee approval was received for this study from the Ethics Committee of Yeditepe University School of Medicine (No: 2018/1518).

Informed Consent: Written informed consent was obtained from the patients who participated in this study.

Acknowledgments: The authors thank Ms. Cigdem Altunok, Department of Biostatistics, Yeditepe University School of Medicine, for her support with the statistical analyses. We also thank Dr. Ayça Başkadem Yılmazer, Department of Otorhinolaryngology, Okmeydanı Training and Research Hospital, for demonstrating the Two Hands Model.

Peer-review: Externally peer-reviewed.

Author Contributions: Concept - R.Y.; Design - R.Y., O.M.T.; Supervision - R.Y., O.M.T.; Data Collection and/or Processing O.M.T.; Analysis and/or Interpretation - R.Y., O.M.T.; Literature Search - R.Y.; Writing - R.Y., O.M.T.; Critical Reviews - R.Y., O.M.T.

Conflict of Interest: The authors have no conflicts of interest to declare.

Financial Disclosure: The authors declared that this study has received no financial support.

\section{References}

1. You P, Instrum R, Parnes L. Benign paroxysmal positional vertigo. Laryngoscope Investig Otolaryngol 2018; 4: 116-23. [CrossRef]

2. Teixido M, Woods O, Kung B, Seyyedi M. A 3D benign paroxysmal positional vertigo model for study of otolith disease. World J Otorhinolaryngol Head Neck Surg 2016; 2: 1-6. [CrossRef]

3. Wang H, Northrop C, Burgess B, Liberman MC, Merchant SN. Three-dimensional virtual model of the human temporal bone: a stand-slone, downloadable teaching tool. Otol Neurotol 2006; 27: 452-7. [CrossRef]

4. Rivas RD.3D didactic model and useful guide of the semicircular conducts. Braz J Otorhinolaryngol 2011; 77: 303-7. [CrossRef]

5. Suzuki R, Taniguchi N, Uchida F, Ishizawa A, Kanatsu Y, Zhou $\mathrm{M}$, et al. Transparent model of temporal bone and vestibulocochlear organ made by 3D printing. Anat Sci Int 2018; 93: 154-9. [CrossRef]

6. Lee ST, Mori Y, Minami K, An CH, Park JW, Kwon TG. Does skeletal surgery for asymmetric mandibular prognathism influence the soft tissue contour and thickness? J Oral Maxillofac Surg 2013; 71: 1577-87. [CrossRef]

7. Aoki S, Takei Y, Suzuki K, Masukawa A, Arai Y. Planer orientation of the bilateral semicircular canals in dizzy patients. Auris Nasus Larynx 2012; 39: 451-4. [CrossRef]

8. Lyu HY, Chen KG, Yin DM, Hong J, Yang L, Zhang TY, et al. The age-related orientational changes of human semicircular canals. Clin Exp Otorhinolaryngol 2016; 9: 109-15. [CrossRef]

9. Bromwich M, Hughes B, Raymond M, Sukerman S, Parnes L. Efficacy of a new home treatment device for benign paroxysmal positional vertigo. Arch Otolaryngol Head Neck Surg 2010; 136: 682-5. [CrossRef]

10. Kim DK, Kim DR, Jeong SH, Kim GJ, Chang KH, Jun BC, Analysis of the coplanarity of functional pairs of semicircular canals using three-dimensional images reconstructed from temporal bone magnetic resonance imaging. J Laryngol Otol 2015; 129: 430-4. [CrossRef]

11. Hashimoto S, Naganuma H, Tokumasu K, Itoh A, Okamoto M. Three-dimensional reconstruction of the human semicircular canals and measurement of each membranous canal plane defined by Reid's stereotactic coordinates. Ann Otol Rhinol Laryngol 2005; 114: 934-8. [CrossRef] 
12. Della Santina CC, Potyagaylo V, Migliaccio AA, Minor LB, Carey JP. Orientation of human semicircular canals measured by three-dimensional multiplanar CT reconstruction. J Assoc Res Otolaryngol 2005; 6: 191-206. [CrossRef]

13. Bradshaw AP, Curthoys IS, Todd MJ, Magnussen JS, Taubman DS, Aw ST, et al. A mathematical model of human semicircular canal geometry: a new basis for interpreting vestibular physiology. J Assoc Res Otolaryngol 2010; 11: 145-59. [CrossRef]

14. Lysakowski A. Anatomy of the Vestibular System. Flint PW, Haughey BH, Lund VJ, Niparko JK, Richardson MA, Robbins KT, et al. editors. Cummings Otolaryngology-Head \& Neck Surgery. 5th ed. Philadelphia: Elsevier Saunders; 2015. p.2007-26. 